International Mathematical Forum, 2, 2007, no. 61, 3013 - 3020

\title{
The Convergence Theorems for Two Nonexpansive Random Operators
}

\author{
Si-Sheng Yao and Lin Wang \\ Department of Mathematics \\ Kunming Teachers College \\ Kunming, Yunnan, 650031, P. R. China \\ yaosisheng@yahoo.com.cn
}

\begin{abstract}
In this paper, we concerned with the study of a random iterative scheme involving two nonexpansive random operators. Using the random iterative scheme, we approximate the random common fixed points of these two operators in a uniformly convex separable Banach space under some appropriate conditions.
\end{abstract}

Mathematics Subject Classification: 47H09, 47J05, 47J25

Keywords: Common random fixed point; Condition (A'); Nonexpansive random operator; Uniformly convex separable Banach space

\section{Introduction.}

Random nonlinear analysis is an important mathematical discipline which is mainly concerned with the study of random nonlinear operators and their properties and is much needed for the study of various classes of random equations. Of course famously random methods have revolutionized the financial markets. Random fixed point theorems for random contraction mappings on separable complete metric spaces were first proved by Spacek [14] and Hans $[7,8]$. The survey article by Bharucha-Reid [1] in 1976 arrtacted the attention of several mathematician and gave wings to this theory. Itoh [9] extended Spacek's and Hans's theorem to multivalued contraction mapping. Now this theory has become the full fledged research area and various ideas associated with random fixed point theory are used to obtain the solution of nonlinear random system(see [2]). Recently Xu [18], Xu and Beg [19], Beg and Shahzad [4] and many other authors have studied the random fixed points of random operator. Mann [12], Outlaw [13], Ishikawa [9,10], and Ghosh and Debnath [6] 
had used different iteration processes to obtain fixed points in deterministic operator theory. The aims of this paper are to study random Ishikawa iterative schemes and to show that the random iterative scheme converges to a random common fixed point of two nonexpansive random operators on the setting of uniformly convex separable Banach space.

\section{Preliminaries}

Let $(\Omega, \Sigma)$ be a measurable space ( $\Sigma$-Sigma algebra) and $C$ a nonempty subset of a Banach space $X$. A mapping $\xi: \Omega \rightarrow X$ is measurable if $\xi^{-1}(U) \in \Sigma$ for each open subset $U$ of $X$. A mapping $T: \Omega \times C \rightarrow C$ is a random map if and only if for each fixed $x \in C$, the mapping $T(\cdot, x): \Omega \rightarrow C$ is measurable, and it is continuous if for each $\omega \in \Omega$, the mapping $T(\omega, \cdot): C \rightarrow X$ is continuous. A measurable mapping $\xi: \Omega \rightarrow X$ is a random fixed point of a random map $T: \Omega \times C \rightarrow X$ if $T(\omega, \xi(\omega))=\xi(\omega)$ for each $\omega \in \Omega$.

Throughout this paper the random mapping $I: \Omega \times C \rightarrow C$ defined by $I(\omega, x)=x$ denotes by $I$, the set of all random fixed points of $T$ denotes by $R F(T)$, respectively.

Definition 2.1. Let $C$ be a nonempty subset of a separable Banach space $X$ and $T: \Omega \times C \rightarrow C$ be a random operator. $T$ is said to be random nonexpansive operator if for arbitrary $x, y \in C$

$$
\|T(\omega, x)-T(\omega, y)\| \leq\|x-y\|
$$

for each $\omega \in \Omega$.

Definition 2.2. Let $X$ be a separable Banach space, $C$ a nonempty subset of $X$. Suppose that $T: \Omega \times C \rightarrow C$ is a random operator. Then

(1) $T$ is said to be demiclosed random operator (at $y)$ if $\left\{x_{n}\right\}$ and $\left\{y_{n}\right\}$ are two sequences such that $T\left(\omega, x_{n}\right)=y_{n}$ and $\left\{x_{n}\right\}$ converges weakly to $x$ and $\left\{T\left(\omega, x_{n}\right)\right\}$ converges to $y$ imply that $x \in C$ and $T(\omega, x)=y$, for each $\omega \in \Omega$.

(2) $T$ is said to be completely continuous random operator if the sequence $\left\{x_{n}\right\}$ in $C$ converges weakly to $x_{0}$ implies that $\left\{T\left(\omega, x_{n}\right)\right\}$ converges strongly to $T\left(\omega, x_{0}\right)$ for each $\omega \in \Omega$.

Definition 2.3. Let $S, T: \Omega \times C \rightarrow C$ be two random operators, where $C$ is nonempty closed and convex subset of a separable Bananch space $X$. Then the random Ishikawa iterative process is defined as follows: for any given measurable mapping $x_{0}: \Omega \rightarrow C$,

$$
\begin{aligned}
y_{n}(\omega) & =b_{n} T\left(\omega, x_{n}(\omega)\right)+\left(1-b_{n}\right) x_{n}(\omega) \\
x_{n+1}(\omega) & =a_{n} S\left(\omega, y_{n}(\omega)\right)+\left(1-a_{n}\right) x_{n}(\omega) \quad n \geq 0
\end{aligned}
$$

for each $\omega \in \Omega$, where $0 \leq a_{n}, b_{n}<1$. 
Remark 1.1. Let $C$ be a closed and convex subset of a separable Banach space $X$ and the sequence of mappings $\left\{x_{n}\right\}$ defined as in Definition 2.3 be pointwise convergent, that is, $x_{n}(\omega) \rightarrow q:=x(\omega)$ for each $\omega \in \Omega$. Then closedness of $C$ implies that $x$ is a mapping from $\Omega$ to $C$. Since $C$ is a subset of a separable Banach space $X$, so, if $S, T$ are two continuous random operators then by [Lemma 8.2.3 of [5]], the map $\omega \rightarrow T(\omega, f(\omega))$ and $\omega \rightarrow S(\omega, f(\omega))$ are measurable mappings for any measurable mapping $f$ from $\Omega$ to $C$. Thus $\left\{x_{n}\right\}$ is a sequence of measurable mappings. Hence $x: \Omega \rightarrow C$, the limit of the sequence of measurable mappings $\left\{x_{n}\right\}$, is also measurable.

For approximating fixed points of deterministic nonexpansive mappings, Senter and Dotson [16] introduced a Condition (A). Later on, Maiti and Ghosh [11], Tan and $\mathrm{Xu}$ [17] studied the Condition (A) and pointed out that Condition (A) is weaker than the requirement of demicompactness.

Definition 2.4. A mapping $T: C \rightarrow C$ is said to satisfy condition (A) if there exists a nondecreasing function $f:[0,+\infty) \rightarrow[0,+\infty)$ with $f(0)=$ $0, f(r)>0$ for all $r \in(0,+\infty)$ such that $\|x-T x\| \geq f(d(x, F(T)))$ for all $x \in C$ where $d(x, F(T))=\inf \left\{\left\|x-x^{*}\right\|: x^{*} \in F(T)\right\}, F(T)$ is the fixed point set of $T$.

For two random operators, we modify the Definition 2.4 as follows.

Definition 2.5. The random operators $S, T: \Omega \times C \rightarrow C$ are said to satisfy condition $\left(\mathrm{A}^{\prime}\right)$ if there exists a nondecreasing function $f:[0,+\infty) \rightarrow$ $[0,+\infty)$ with $f(0)=0, f(r)>0$ for all $r \in(0,+\infty)$ such that $\frac{1}{2}(\|x-T(\omega, x)\|+$ $\|x-S(\omega, x)\|) \geq f\left(d\left(x, F_{1}\right)\right)$ for all $x \in C$ and each $\omega \in \Omega$, where $F_{1}=$ $R F(S) \bigcap R F(T)$ and $d\left(x, F_{1}\right)=\inf \left\{\left\|x-x^{*}\right\|: x^{*} \in F_{1}\right\}$.

We restate the following lemmas which play important roles in our proofs.

Lemma 2.1 [16]. Suppose that $X$ is a uniformly convex Banach space and $0<p \leq t_{n} \leq q<1$ for all positive integers $n$. Also suppose that $\left\{x_{n}\right\}$ and $\left\{y_{n}\right\}$ are two sequences in $X$ such that $\limsup _{n \rightarrow \infty}\left\|x_{n}\right\| \leq r, \quad \limsup _{n \rightarrow \infty}\left\|y_{n}\right\| \leq r$ and $\limsup _{n \rightarrow \infty}\left\|t_{n} x_{n}+\left(1-t_{n}\right) y_{n}\right\|=r$ hold for some $r \geq 0$. Then $\lim _{n \rightarrow \infty} \| x_{n}-$ $y_{n} \|=0$.

Lemma 2.2 [3]. Let $X$ be a real uniformly convex Banach space, $C$ a nonempty closed convex subset of $X$, and let $T: C \rightarrow X$ be a deterministic nonexpansive mapping. Then $I-T$ is demiclosed at zero.

\section{Convergence of the iteration scheme.}

Lemma 3.1. Let $X$ be a uniformly convex separable Banach space, $C$ a nonempty closed convex subset of $X$. Let $S, T: \Omega \times C \rightarrow C$ be two random nonexpansive operators. Suppose sequence $\left\{x_{n}\right\}$ is generated by $(2.1)$. If $F_{1} \neq \emptyset$, then $\lim _{n \rightarrow \infty}\left\|x_{n}(\omega)-x^{*}(\omega)\right\|$ exists for any $x^{*} \in F_{1}=R F(S) \cap R F(T)$ and each $\omega \in \Omega$. 
Proof. For any $x^{*} \in F_{1}$, then for each $\omega \in \Omega$, we have

$$
\begin{aligned}
0 & \leq\left\|x_{n+1}(\omega)-x^{*}(\omega)\right\|=\left\|a_{n} S\left(\omega, y_{n}(\omega)\right)+\left(1-a_{n}\right) x_{n}(\omega)-x^{*}(\omega)\right\| \\
& =\left\|a_{n}\left(S\left(\omega, y_{n}(\omega)\right)-x^{*}(\omega)\right)+\left(1-a_{n}\right)\left(x_{n}(\omega)-x^{*}(\omega)\right)\right\| \\
& \leq a_{n}\left\|S\left(\omega, y_{n}(\omega)\right)-S\left(\omega, x^{*}(\omega)\right)\right\|+\left(1-a_{n}\right)\left\|x_{n}(\omega)-x^{*}(\omega)\right\| \\
& \leq a_{n}\left\|y_{n}(\omega)-x^{*}(\omega)\right\|+\left(1-a_{n}\right)\left\|x_{n}(\omega)-x^{*}(\omega)\right\| \\
& =a_{n}\left\|b_{n} T\left(\omega, x_{n}(\omega)\right)+\left(1-b_{n}\right) x_{n}(\omega)-x^{*}(\omega)\right\|+\left(1-a_{n}\right)\left\|x_{n}(\omega)-x^{*}(\omega)\right\| \\
& =a_{n}\left\|b_{n}\left(T\left(\omega, x_{n}(\omega)\right)-x^{*}(\omega)\right)+\left(1-b_{n}\right)\left(x_{n}(\omega)-x^{*}(\omega)\right)\right\|+\left(1-a_{n}\right)\left\|x_{n}(\omega)-x^{*}(\omega)\right\| \\
& \leq a_{n} b_{n}\left\|T\left(\omega, x_{n}(\omega)\right)-T\left(\omega, x^{*}(\omega)\right)\right\|+a_{n}\left(1-b_{n}\right)\left\|x_{n}(\omega)-x^{*}(\omega)\right\|+\left(1-a_{n}\right)\left\|x_{n}(\omega)-x^{*}(\omega)\right\| \\
& \leq a_{n} b_{n}\left\|x_{n}(\omega)-x^{*}(\omega)\right\|+a_{n}\left(1-b_{n}\right)\left\|x_{n}(\omega)-x^{*}(\omega)\right\|+\left(1-a_{n}\right)\left\|x_{n}(\omega)-x^{*}(\omega)\right\| \\
& =\left\|x_{n}(\omega)-x^{*}(\omega)\right\| .
\end{aligned}
$$

Thus the sequence $\left\{\left\|x_{n}(\omega)-x^{*}(\omega)\right\|\right\}$ is decreasing and hence $\left\{x_{n}(\omega)\right\}$ is bounded. So, $\lim _{n \rightarrow \infty}\left\|x_{n}(\omega)-x^{*}(\omega)\right\|$ exists for each $x^{*} \in F_{1}$, for each $\omega \in \Omega$. The proof is completed.

Lemma 3.2. Let $X$ be a uniformly convex separable Banach space and $C$ a nonempty closed convex subset of $X$. Let $S, T: \Omega \times C \rightarrow C$ be two nonexpansive random operators. Suppose $\left\{x_{n}\right\}$ is generated by $(2.1)$. If $F_{1} \neq \emptyset$, then $\lim _{n \rightarrow \infty}\left\|S\left(\omega, x_{n}(\omega)\right)-x_{n}(\omega)\right\|=\lim _{n \rightarrow \infty}\left\|T\left(\omega, x_{n}(\omega)\right)-x_{n}(\omega)\right\|=0$, for each $\omega \in \Omega$.

Proof. By Lemma 3.1, for any $x^{*} \in F_{1}, \lim _{n \rightarrow \infty}\left\|x_{n}(\omega)-x^{*}(\omega)\right\|$ exists, for each $\omega \in \Omega$. For given $\omega \in \Omega$, assume $\lim _{n \rightarrow \infty}\left\|x_{n}(\omega)-x^{*}(\omega)\right\|=c \geq 0$. Since

$$
\begin{aligned}
\left\|y_{n}(\omega)-x^{*}(\omega)\right\| & =\left\|b_{n}\left(T\left(\omega, x_{n}(\omega)\right)-x^{*}(\omega)\right)+\left(1-b_{n}\right)\left(x_{n}(\omega)-x^{*}(\omega)\right)\right\| \\
& \leq b_{n}\left\|T\left(\omega, x_{n}(\omega)\right)-T\left(\omega, x^{*}(\omega)\right)\right\|+\left(1-b_{n}\right)\left\|x_{n}(\omega)-x^{*}(\omega)\right\| \\
\leq & b_{n}\left\|x_{n}(\omega)-x^{*}(\omega)\right\|+\left(1-b_{n}\right)\left\|x_{n}(\omega)-x^{*}(\omega)\right\| \\
& =\left\|x_{n}(\omega)-x^{*}(\omega)\right\| .
\end{aligned}
$$

Taking limsup on both sides in above inequality, we have

$$
\limsup _{n \rightarrow \infty}\left\|y_{n}(\omega)-x^{*}(\omega)\right\| \leq c .
$$

Noticing,

$$
\left\|S\left(\omega, y_{n}(\omega)\right)-x^{*}(\omega)\right\|=\left\|S\left(\omega, y_{n}(\omega)\right)-S\left(\omega, x^{*}(\omega)\right)\right\| \leq\left\|y_{n}(\omega)-x^{*}(\omega)\right\| .
$$

Taking limsup on both sides in the above inequality and then using (3.1), we have

$$
\limsup _{n \rightarrow \infty}\left\|S\left(\omega, y_{n}(\omega)\right)-x^{*}(\omega)\right\| \leq c .
$$


Further, $\lim _{n \rightarrow \infty}\left\|x_{n+1}(\omega)-x^{*}(\omega)\right\|=c$ means that

$$
\lim _{n \rightarrow \infty}\left\|a_{n}\left(S\left(\omega, y_{n}(\omega)\right)-x^{*}(\omega)\right)+\left(1-a_{n}\right)\left(x_{n}(\omega)-x^{*}(\omega)\right)\right\|=c .
$$

Hence applying Lemma 2.1, we obtain that

$$
\lim _{n \rightarrow \infty}\left\|S\left(\omega, y_{n}(\omega)\right)-x_{n}(\omega)\right\|=0
$$

Next,

$$
\begin{aligned}
\left\|x_{n}(\omega)-x^{*}(\omega)\right\| & \leq\left\|x_{n}(\omega)-S\left(\omega, y_{n}(\omega)\right)\right\|+\left\|S\left(\omega, y_{n}(\omega)\right)-x^{*}(\omega)\right\| \\
& \leq\left\|x_{n}(\omega)-S\left(\omega, y_{n}(\omega)\right)\right\|+\left\|y_{n}(\omega)-x^{*}(\omega)\right\|
\end{aligned}
$$

gives that $c \leq \operatorname{limin} f_{n \rightarrow \infty}\left\|y_{n}(\omega)-x^{*}(\omega)\right\| \leq \limsup _{n \rightarrow \infty}\left\|y_{n}(\omega)-x^{*}(\omega)\right\| \leq c$. That is $\lim _{n \rightarrow \infty}\left\|y_{n}(\omega)-x^{*}(\omega)\right\|=c$.

Now $\lim _{n \rightarrow \infty}\left\|y_{n}(\omega)-x^{*}(\omega)\right\|=c$ is expressible as

$$
\lim _{n \rightarrow \infty}\left\|b_{n}\left(T\left(\omega, x_{n}(\omega)\right)-x^{*}(\omega)\right)+\left(1-b_{n}\right)\left(x_{n}(\omega)-x^{*}(\omega)\right)\right\|=c .
$$

Since $\lim _{n \rightarrow \infty}\left\|x_{n}(\omega)-x^{*}(\omega)\right\|=c$ and $\limsup _{n \rightarrow \infty}\left\|T\left(\omega, x_{n}(\omega)\right)-x^{*}(\omega)\right\| \leq$ $\limsup _{n \rightarrow \infty}\left\|x_{n}(\omega)-x^{*}(\omega)\right\| \leq c$, hence, it follows from Lemma 2.1 that

$$
\lim _{n \rightarrow \infty}\left\|T\left(\omega, x_{n}(\omega)\right)-x_{n}(\omega)\right\|=0
$$

Now observe that

$$
\begin{aligned}
\| S\left(\omega, x_{n}(\omega)\right)- & x_{n}(\omega)\|\leq\| S\left(\omega, x_{n}(\omega)\right)-S\left(\omega, y_{n}(\omega)\right)\|+\| S\left(\omega, y_{n}(\omega)\right)-x_{n}(\omega) \| \\
\leq & \left\|x_{n}(\omega)-y_{n}(\omega)\right\|+\left\|S\left(\omega, y_{n}(\omega)\right)-x_{n}(\omega)\right\| \\
& =\left\|x_{n}(\omega)-b_{n} T\left(\omega, x_{n}(\omega)\right)-\left(1-b_{n}\right) x_{n}(\omega)\right\|+\left\|S\left(\omega, y_{n}(\omega)\right)-x_{n}(\omega)\right\| \\
& =b_{n}\left\|x_{n}(\omega)-T\left(\omega, x_{n}(\omega)\right)\right\|+\left\|S\left(\omega, y_{n}(\omega)\right)-x_{n}(\omega)\right\| .
\end{aligned}
$$

Taking limsup on both sides in the above inequality, by (3.2) and (3.3) we achieve that

$$
\lim _{n \rightarrow \infty}\left\|S\left(\omega, x_{n}(\omega)\right)-x_{n}(\omega)\right\|=0 .
$$

Hence $\lim _{n \rightarrow \infty}\left\|S\left(\omega, x_{n}(\omega)\right)-x_{n}(\omega)\right\|=\lim _{n \rightarrow \infty}\left\|T\left(\omega, x_{n}(\omega)\right)-x_{n}(\omega)\right\|=0$, for each $\omega \in \Omega$. The proof is completed.

Theorem 3.3. Let $X$ be a uniformly convex separable Banach space and $C, S, T,\left\{x_{n}\right\}$ be same as in Lemma 3.1. If $F_{1} \neq \emptyset$ and $S, T$ satisfy the Condition $\left(\mathrm{A}^{\prime}\right)$, then

(1) $F_{1}$ is a closed set.

(2) $\left\{x_{n}\right\}$ converges to a common random fixed point of $S$ and $T$. 
Proof. (1). Let $\left\{\xi_{n}\right\} \subset F_{1}$ be such that $\xi_{n}(\omega) \rightarrow x(\omega)$ as $n \rightarrow \infty$, for each $\omega \in \Omega$. Since $\left\{\xi_{n}\right\}$ is a measurable sequence, the limit $x$ of $\left\{\xi_{n}\right\}$ is measurable, too. In addition

$\|T(\omega, x(\omega))-x(\omega)\|=\left\|T(\omega, x(\omega))-T\left(\omega, \xi_{n}(\omega)\right)+\xi_{n}(\omega)-x(\omega)\right\| \leq 2\left\|\xi_{n}(\omega)-x(\omega)\right\|$.

This implies that $T(\omega, x(\omega))=x(\omega)$, for each $\omega \in \Omega$, therefore, $x \in R F(T)$. Similarly, we have $x \in R F(S)$. Thus, $x$ is a common random fixed point of $S$ and $T$. Hence $F_{1}$ is a closed set.

(2). For any $x^{*} \in F_{1}$, by Lemma 3.1, for each $\omega \in \Omega, \lim _{n \rightarrow \infty} \| x_{n}(\omega)-$ $x^{*}(\omega) \|$ exists. Let it be $c$ for some $c \geq 0$. If $c=0$, there is nothing to prove.

If $c>0$, by Lemma 3.2, $\lim _{n \rightarrow \infty}\left\|S\left(\omega, x_{n}(\omega)\right)-x_{n}(\omega)\right\|=\lim _{n \rightarrow \infty} \| T\left(\omega, x_{n}(\omega)\right)-$ $x_{n}(\omega) \|=0$, for each $\omega \in \Omega$. Moreover, $\left\|x_{n+1}(\omega)-x^{*}(\omega)\right\| \leq\left\|x_{n}(\omega)-x^{*}(\omega)\right\|$ gives that

$$
i n f_{x^{*} \in F_{1}}\left\|x_{n+1}(\omega)-x^{*}(\omega)\right\| \leq i n f_{x^{*} \in F_{1}}\left\|x_{n}(\omega)-x^{*}(\omega)\right\|
$$

That implies that $0 \leq d\left(x_{n+1}(\omega), F_{1}\right) \leq d\left(x_{n}(\omega), F_{1}\right)$. Thus $\lim _{n \rightarrow \infty} d\left(x_{n}(\omega), F_{1}\right)$ exists, for each $\omega \in \Omega$.

Since $S, T$ satisfy Condition (A'), we have

$$
\frac{1}{2}\left(\left\|x_{n}(\omega)-T\left(\omega, x_{n}(\omega)\right)\right\|+\left\|x_{n}(\omega)-S\left(\omega, x_{n}(\omega)\right)\right\|\right) \geq f\left(d\left(x_{n}(\omega), F_{1}\right)\right)
$$

It follows from (3.2) and (3.3) that we have $\lim _{n \rightarrow \infty} f\left(d\left(x_{n}(\omega), F_{1}\right)\right) \leq 0$. Since $f$ is a nondecreasing function with $f(0)=0$, therefore, $\lim _{n \rightarrow \infty} d\left(x_{n}(\omega), F_{1}\right)=$ 0 , for each $\omega \in \Omega$.

For any $\varepsilon>0$ and any given $\omega \in \Omega$, since $\lim _{n \rightarrow \infty} d\left(x_{n}(\omega), F_{1}\right)=0$, there exists natural number $n_{1}$ such that when $n \geq n_{1}, d\left(x_{n}(\omega), F_{1}\right)<\frac{\varepsilon}{3}$. Thus, there exists $x^{*} \in F_{1}$ such that for above $\varepsilon$ and $\omega$ there exists positive integer $N_{1} \geq n_{1}$ such that as $n \geq N_{1}$

$$
\left\|x_{n}(\omega)-x^{*}(\omega)\right\|<\frac{\varepsilon}{2} .
$$

Now for arbitrary $n, m \geq N_{1}$, consider

$$
\left\|x_{n}(\omega)-x_{m}(\omega)\right\| \leq\left\|x_{n}(\omega)-x^{*}(\omega)\right\|+\left\|x_{m}(\omega)-x^{*}(\omega)\right\|<\frac{\varepsilon}{2}+\frac{\varepsilon}{2}=\varepsilon .
$$

It implies that $\left\{x_{n}(\omega)\right\}$ is a cauchy sequence in $C$. Let $x_{n}(\omega) \rightarrow x(\omega)$. Since $\left\{x_{n}\right\}$ is a sequence of measurable mappings, so $x$, the limit of the sequence of measurable mapping $\left\{x_{n}\right\}$, is measurable, too. In addition, it follows from Lemma 2.2 and Lemma 3.2 that $T(\omega, x(\omega))=x(\omega)=S(\omega, x(\omega))$. Therefore, $x$ is a common random fixed point of $S$ and $T$. Thus, by Lemma 3.1, $\lim _{n \rightarrow \infty}\left\|x_{n}(\omega)-x(\omega)\right\|=0$, for each $\omega \in \Omega$. This implies that $\left\{x_{n}\right\}$ converges to the common random fixed point $x$ of $S$ and $T$. The proof is completed. 
Theorem 3.4. Let $X$ be a uniformly convex separable Banach space and $C, S, T,\left\{x_{n}\right\}$ be same as in Lemma 3.1. If $F_{1} \neq \emptyset$, one of $S$ and $T$ is completely continious random operator, then $\left\{x_{n}\right\}$ converges to a common random fixed point of $S$ and $T$.

Proof. For any $x^{*} \in F_{1}$, by Lemma 3.1, $\lim _{n \rightarrow \infty}\left\|x_{n}(\omega)-x^{*}(\omega)\right\|$ exists for each $\omega \in \Omega$.

By the condition of Theorem 3.4, we may assume that $T$ is completely continuous random operator. Since, $\left\{x_{n}(\omega)\right\}$ is a bounded sequence and $X$ is uniformly convex separable Banach space, there exists convergent subsequence $\left\{x_{n_{k}}(\omega)\right\}$ of $\left\{x_{n}(\omega)\right\}$ such that $\left\{T\left(\omega, x_{n_{k}}(\omega)\right)\right\}$ is convergent, for each $\omega \in \Omega$. Let $\left\{x_{n_{k}}(\omega)\right\} \rightarrow \xi(\omega)$ for each $\omega \in \Omega$, then $T(\omega, \xi(\omega))=\xi(\omega)$ for each $\omega \in \Omega$. The mapping $\xi: \Omega \rightarrow C$ being a pointwise limit of the measurable mappings $\left\{x_{n}(\omega)\right\}$, is measurable, by the continuity of $T$ and (3.3). That is $\xi \in R F(T)$

By (3.4) and $x_{n_{k}}(\omega) \rightarrow \xi(\omega)$ as $k \rightarrow \infty$, we have $S(\omega, \xi(\omega))=\xi(\omega)$, for each $\omega \in \Omega$. So, $\xi \in R F(S)$. Hence $\xi \in F_{1}$.

Since $\lim _{n \rightarrow \infty}\left\|x_{n}(\omega)-\xi(\omega)\right\|$ exists and $\lim _{k \rightarrow \infty}\left\|x_{n_{k}}(\omega)-\xi(\omega)\right\|=0$ for each $\omega \in \Omega$. Therefore, $\lim _{n \rightarrow \infty}\left\|x_{n}(\omega)-\xi(\omega)\right\|=0$, for each $\omega \in \Omega$. The proof is completed.

Acknowledgement. This work was supported by the Scientific Research Foundation of Kunming Teachers College (2007z002).

\section{References}

[1] A.T. Bharucha-Reid, Fixed point theorems in probabilistic analysis, Bull. Amer. Math. Soc. 82, (1976), 641-657.

[2] A.T. Bharucha-Reid, Random Integral Equations, Academic Press, New York, 1972.

[3] F.E. Browder, Semicontractive and semi accretive nonlinear mappings in Banach spaces, Bull. Amer. Math. Soc. 74, (1968), 660-665.

[4] I. Beg, N. Shahzad, Random fixed point theorems for nonexpansive and contractive type random operators on Banach spaces, J. Appl. Math. Stochastic Anal. 7, (1994), 569-580.

[5] M.K. Ghosh, L. Debnath, Convergence of Ishikawa iterates of quasinonexpansive mappings, J. Math. Anal. Appl. 207(1997)96-103.

[6] O. Hans, Reduzierende zulliăllige transformaten, Czechoslovak Math. J. 7, (1957), 154-158. 
[7] O. Hans, Random operator equations, in: Proceedings of the Fourth Berkeley Symposium on Mathematical Statistics and Probability, vol. II, Part I, University of Califormia Press, Berkeley, 1961, pp. 85-202.

[8] S. Itoh, Random fixed point theorems with an application to random differential equations in Banach spaces, J. Math. Anal. Appl. 67, (1979), 261-273.

[9] S. Ishikawa, Fixed points by a new iteration method, Proc. Amer. Math. Soc. 44, (1974), 147-150.

[10] S. Ishikawa, Fixed points and iteration of a nonexpansive mapping in a Banach space, Proc. Amer. Math. Soc. 59, (1976), 65-71.

[11] M. Maiti, M.K. Gosh, Approximating fixed points by Ishikawa iterates, Bull. Austral. Math. Soc. 40, (1989), 113-117.

[12] W.R. Mann, Mean valued methods in iteration, Proc. Amer. Math. Soc. 4, (1953), 506-510.

[13] C.L. Outlaw, Mean value iteration of nonexpansive mappings in Banach spacces, Pacific J. Math. 30, (1969), 747-750.

[14] A. Spacek, Zufállige gleichungen, Czechoslovak Math. J. 5, (1995), 462466.

[15] H.F. Senter, W.G. Dotson, Approximating fixed points of nonexpansie mappings, Proc. Amer. Math. Soc. 44(2), (1974), 375-380.

[16] J. Schu, Weak and strong convergence to fixed points of nonexpansive mappings, Bull. Austral. Math. Soc. 43, (1991), 153-159.

[17] K.K. Tan, H.K. Xu, Approximating fixed points of nonexpansive mapings by the Ishikawa iteration process, J. Math. Anal. Appl. 178, (1993), 301308.

[18] H.K. Xu, Some random fixed point theorems for condensing and nonexpansive operators, Proc. Amer. Math. Soc. 110, (1990), 103-123.

[19] H.K. Xu, I. Beg, Measurability of fixed point sets of multivalued random operators, J. Math. Anal. Appl. 225, (1998), 62-72.

\section{Received: April 4, 2007}

